Aus der I. Medizinischen Klinik (Direktor: Prof. Dr. H. Квозсн)

der Martin-Luther-Universität, Halle-Wittenberg

\title{
Schweizer Leopoldina-Mitglieder des 17. und 18. Jahrhunderts und ihre Korrespondenz mit den Akademiepräsidenten
}

Von Wolfram Kaiser und Arina Völker

Die am 1. Januar 1652 gegründete Academica Naturae Curiosorum, die älteste deutsche naturwissenschaftliche Akademie der Gegenwart, hat bis zum Ende des 18. Jahrhunderts mehr als vierzig Schweizer Mitglieder in ihre Reihen aufgenommen. Das ist eine stattliche Zahl, die sogar noch vergrößert werden könnte, wenn man die in den Periodica der Gesellschaft publizierenden Ärzte und Naturforscher hinzurechnet; zweifellos war auch bei letzteren im einen oder anderen Falle eine Rezeption vorgesehen, unterblieb dann aber aus heute der Nachprüfbarkeit entzogenen Gründen.

Die genannten Mitgliedschaften in einem Zeitraum von 150 Jahren konzentrieren sich besonders auf die Präsidialtätigkeit von Johann Michael Fehr (1610-1688) in Schweinfurt, Johann Georg Volckamer (1616-1693) in Nürnberg und Lucas Schroeck (1646-1730) in Augsburg; Fehr hat in den Jahren 1666 bis 1686, Volckamer im Anschluß bis 1693 und Schroeck schließlich bis 1730 den Vorsitz dieser hochangesehenen Sozietät inne. In fünf Dezennien kommt es hierbei zu 26 Schweizer Berufungen, denen eine wesentlich geringere Berufungszahl in dem übrigen hier abgehandelten Zeitraum gegenübersteht. So darf man Fehr, Volckamer und Schroeck wohl eine besondere Aufgeschlossenheit gegenüber den Wissenschaftskontakten zur benachbarten Schweiz attestieren, was auch aus der erhalten gebliebenen Korrespondenz jener Epoche zu belegen ist und zusätzlich in einer regen publizistischen Aktivität der betreffenden Mitglieder zum Ausdruck kommt. Die Tatsache, daß die Leitungstätigkeit der drei genannten Leopoldina-Präsidenten in eine Blüteperiode der Schweizer medizinischen und naturwissenschaftlichen Forschung fällt, mag dieses rege Engagement zusätzlich erklären.

Das Briefarchiv der Gesellschaft, die heute unter dem Namen «Deutsche Akademie der Naturforscher Leopoldina» fortbesteht, enthält eine Reihe bislang noch wenig bekannter und zum Teil sogar unerschlossener Doku- 
mentationen, auf die mit diesem Beitrag hingewiesen werden soll. Dabei wurde in diesem Zusammenhang bewußt auf ausführliche biographische Darstellungen verzichtet; Leben und Wirken der Schweizer LeopoldinaMitglieder wurden nur so weit gestreift, wie es sich im Rahmen der aufzuzeigenden Korrespondenz als erforderlich erwies.

Die beiden ersten hier zu nominierenden Persönlichkeiten sind zwei Ärzte, die zwar nicht aus der Schweiz gebürtig sind, durch ihren Berufsweg aber eine Rolle in der Berner Medizingeschichte spielen: der Stadtphysikus Johann David Wilhelmi (1619-1671) und der Fakultätsassessor JohanN ЈАков W iтtig (1634-1663). Beide werden unter dem als I. Präses fungierenden Johann Lorenz Bausch (1605-1665) in die Akademie berufen: der aus Bacharach gebürtige Wilhelmi am 30. Dezember 1652, der aus Erfurt stammende Wittig am 29. Juli 1662. Ein Schriftverkehr mit der Leopoldina ist in deren Archiv nicht mehr erhalten.

Ab 1681 setzt dann eine rege Rezeption Schweizer Kandidaten ein: innerhalb eines Dezenniums kommen elf Ärzte aus Basel, Schaffhausen, Dießenhofen und Zürich zur Aufnahme.

Die Schweizer Berufungen in der Amtsperiode von J.M. Fehr und J.G. Volckamer

erreichen in den Jahren 1681 bis 1691 einen ersten Kulminationspunkt: laufend erscheinen jetzt die Namen prominenter Schweizer Ärzte in den Aufnahmeartikeln der Gesellschaft. Vorwiegend sind es die Vertreter der berühmten Schaffhauser Ärzteschule (22), deren Verdienste um die Anatomie und Physiologie sowie generell um die experimentelle Medizin hier kaum der erneuten Betonung bedürfen. In der Reihenfolge der Aufnahme sind es in der Fehrschen Ära die Ärzte Johann Jaков Harder (1656-1711), Johann Conrad Peyer (1653-1712), Johann von Muralt (1645-1733), Heinrich Screta (1637-1689), Emmanuel König (1658-1731), Johann Jakob Wepfer (1620-1695), Johann Conrad Brunner (1653-1727) und Theodor Zwinger (1658-1724). Johann Georg Volckamer nimmt Joseph Müller (1659-1711), JohanN Jakob Wagner (1641-1695) und SyLvester Samuel Anhorn von Hartwiss in die Sozietät auf.

Für den Nachbetrachter wirkt es überraschend, daß nicht Johann Jakob Wepfer als Altmeister der Schaffhauser Ärzteschule, sondern zunächst seine Kollegen und Mitarbeiter Harder und Peyer am 14. Juli 1681 die Berufungsliste einleiten. Der später durch seine pathologisch-anatomischen 
Abhandlungen hervorgetretene Harder ist zum Zeitpunkt seiner Rezeption wissenschaftlich noch nicht so hervorragend ausgewiesen, daß ihm diese Vorzugsstellung zugekommen wäre. Er verdankt die frühe Berufung wohl den guten Beziehungen zu seinem Korrespondenzpartner Johann Wolfgang Wedel (1645-1721) in Jena, der als Adjunkt der Gesellschaft fungiert (18). Der auf einer Professur in Basel wirkende Harder hatte im gleichen Jahr eine teratologische Arbeit an Wedel abgeschickt, der sich nun wohlwollend beim amtierenden Präsidenten für den neuen Kandidaten einsetzte.

Es wäre aber sicher ungerecht, lediglich Harders «Beziehungen» und die hieraus resultierende Ehrung vordergründig sehen zu wollen: schon sein Briefwechsel des Jahres 1681 mit dem Akademiepräsidenten Fehr weist aus, daß es ihm hier um eine zweckgebundene Korrespondenz mit dem Ziel des wissenschaftlichen Gedankenaustausches geht. Als Harder nämlich im Anschluß an seine Aufnahme das für jedes Mitglied obligatorische Curriculum vitae einreicht, führt er darin unter der Überschrift «Nomina Viror. Clariss. quibuscum per literas confabulor» eine Rubrik des Personenkreises auf, mit dem er in schriftlicher Verbindung steht. Er nominiert in dieser Aufstellung nächst Wedel u. a. Georg Franck (1644-1704) in Heidelberg, Johann Jakob Waldschmidt (1644-1687) in Marburg sowie die späteren Leopoldina-Präsidenten Volckamer und Schroeck; von seinen Schweizer Landsleuten nennt er im gleichen Zusammenhang die Ärzte Wepfer, Screta, Wagner und Brunner. Schließlich gibt er in diesem Schreiben der Akademie Nachricht über seine bisherigen Forschungsergebnisse und verspricht eine regelmäßige Mitarbeit für die Miscellanea:

«Prae ceteris autem Anatomes culturam meas dixerim delitias, et quasi Anatomicum sane me pronuntiens (absit inanis talis gloriosa) sed quod exhinc maximam in re medica utilitatem mihi accessisse, fateri necesse habeo ... O' Curiosi! conscribere medebor!» (1).

Mit der Einhaltung dieses Versprechens hat es aber dann wohl doch Schwierigkeiten gegeben; die Basler Praxis nimmt Harder jedenfalls stark in Anspruch, und ohne seine regelmäßigen Schaffhauser Aufenthalte wäre Harder wohl kaum noch zu wissenschaftlichen Resultaten gekommen. Für die Akademie scheint seine Mittlerrolle aber stets von Bedeutung geblieben zu sein: 1689 wird er zu deren Adjunkt ernannt. Einem Brief Harders an das Akademiesekretariat ist zu entnehmen, daß z. B. Johann Georg Joseph Schwaller aus Solothurn die Aufnahme in die Leopoldina dem Vorschlag seines Landsmannes verdankt. 
Der gleichzeitig mit Harder in die Akademie berufene Johann Conrad Peyer aus Schaffhausen hatte schon am 8. Juni 1681 - also noch vor der offiziellen Aufnahme - sein Curriculum vitae an die Gesellschaft eingereicht. Der durch seine Arbeiten über die Intestinaldrüsen hervorgetretene Arzt steht ebenfalls seit langem in Kontakt zu Volckamer und Schroeck; hinsichtlich seiner wissenschaftlichen Ausrichtung beruft er sich auf seine Schaff hauser Lehrer Johann Jakob Wepfer, Heinrich Screta und Christoph Harder (1625-1689), wobei er im einzelnen ausführt:

«... postquam aliud biennium inter anatomica et chemica experimenta transegissem atque inprimis celebrium medicorum trium, Johannis Jacobi Wepferi, Christophori Harderi, et Henrici Scretae, consuetudine usus fuissem, horum praecipue suasu atque hortatu ad reddendam otii rationem de glandulis intestinorum et ventriculo gallinaceo exercitationem divulgavi» (2).

Am 5. November 1681 bedankt sich Peyer bei Fehr für die inzwischen erfolgte Aufnahme und das Cognomen «Pythagoras»; sein Freund Harder hat den Beinamen «Paeon» erhalten:

«Nam academia vestra donatum esse me et Pythagoram audire felicitatis mundanae summa est laetitiaeque cumulus»(2).

In der Folgezeit entwickelt Peyer, der 1685 seine Merycologia herausbringt $(30,36)$, eine rege Aktivität, weiteren schaffhauserischen, baslerischen und Zürcher Forschern die Mitgliedschaft der Gesellschaft zu vermitteln (22). So legt er mit einem Schreiben vom 15. April 1684 das Curriculum vitae seines Kollegen Joseph Müller vor, den er zur Aufnahme empfiehlt und über den er mitteilt:

«Mylleri brevem mitto historiam vitae. Is recipi quidem gessit ac cooptari in numerum Collegarum Academiae Naturae Curiosorum, fidemque suam promittit, nullum tamen cognomen expetit, donec praestiterit quae leges requirunt» (2).

Nächst Harder und Peyer wird Johann von Muralt am 13. Dezember 1681 Mitglied der Akademie. Der durch seine Bemühungen um die Integrierung der Chirurgie in die wissenschaftliche Medizin hervorgetretene Zürcher Arzt (35) stellt Fehr im Januar 1682 mit einem Dankschreiben sein Curiculum vitae zu:

«Ego Johannes de Muralto Chirurg. et Med.D.Facult. Med.Basil. Assessor, divino favente Numine, novi huius anni auspiciis, in numerum Sanct.Rom. Imp. Naturae Curiosorum adscriptus, ac illustri Aretasi virtuosissimi medici agnomine condecoratus, vitae meae curriculum Archivis inserendum expositurus ...» (3). 
Bei der Aufzählung der umfangreichen Stationen seiner medizinischen Bildungsreise versäumt der 1671 in Basel promovierte Verfasser des Vademecum anatomicum (1677) auch nicht, seines Studien- und Weggefährten Christoph Helvigius (1656-1690) Erwähnung zu tun; gemeinsame botanische Interessen hatten Muralt zusammen mit dem aus Anklam gebürtigen und später als Ordinarius in Greifswald wirkenden Helvigius zu den führenden Horti medici pilgern lassen.

Heinrich Screta von Zovorziz folgt als nächster Schweizer Arzt in den Matrikellisten der Leopoldina. Der Sohn des Prager Exulanten und ab 1635 als Stadtphysikus von Schaffhausen amtierenden Johann Screta war 1670 in Heidelberg promoviert worden. Als wissenschaftlich interessierter Praktiker wirkt er in seiner Wahlheimat, als ihn am 6. Mai 1682 die ehrenvolle Berufung erreicht. Seine schon vom Vater inaugurierte Arbeit über die Lagersucht - Morbus Hungaricus $(19,39)$ - ist erst drei Jahre nach der Rezeption erschienen; sie dürfte in der Schweiz, deren Landeskinder als Söldner auch auf den südosteuropäischen Kriegsschauplätzen kämpften, auf großes Interesse gestoßen sein.

Der am 17. August 1682 rezipierte Emmanuel König lehrt zu diesem Zeitpunkt an der Universität Basel. Er verdankt seine Berufung - das geht aus seinem Dankschreiben mit gleichzeitig eingereichtem Curriculum vitae unmißverständlich hervor - der Empfehlung Wedels; dieser wiederum dürfte durch Harder beraten worden sein. Der botanisch und zoologisch ausgerichtete König legt bei dieser Gelegenheit dem Akademiepräsidenten Fehr eine seiner Buchpublikationen vor: Mitto quoque exemplar Regni mei Animalis (4), scheint sich später aber wenig um die Belange der Sozietät gekümmert zu haben; 1697 mahnt Schroeck auf Umwegen an, König «aus seinem Schlaf aufzuwecken» (18). Genau wie Peyer und Muralt hat König übrigens voll Stolz das Wappen der Leopoldina in seinem eigenen Siegel geführt (37).

Am 11. Mai 1685 wird nun endlich auch Johann Jakob Wepfer in die Akademie berufen. Leben und Werk dieses vor allem um die Anatomie, Physiologie und Toxikologie hochverdienten Arztes sind bekannt (16, 20, 21, 32); seine erhaltengebliebene Korrespondenz wurde aufgezeigt und interpretiert. Im Briefarchiv der Leopoldina fehlen heute leider jedwelche Unterlagen über einen zweifellos vorhanden gewesenen wissenschaftlichen Gedankenaustausch; das gilt auch für Wepfers zum gleichen Zeitpunkt aufgenommenen Schwiegersohn Johann Conrad Brunner. Der aus DieBenhofen gebürtige Professor primarius in Heidelberg - als «von Brunn, 
Dominus in Hammerstein » nobiliert - nimmt durch seine experimentellen Untersuchungen über die Pankreasfunktion einen bedeutsamen Platz in der Geschichte der Physiologie und speziell auch der Diabetologie ein (40).

Schließlich kommt zum gleichen Zeitpunkt mit Theodor Zwinger ein Vertreter der bekannten Basler Anatomendynastie zur Aufnahme. Zwinger hatte sich vorübergehend zur Ausbildung bei Wepfer in Schaffhausen aufgehalten, und so ist die gemeinsame Berufung des Lehrers und seiner Schüler wahrscheinlich aus der Initiative des Schaffhauser Forscherkreises zu erklären. Leider ist auch sein Curriculum vitae bei der Akademie nicht erhalten geblieben; auf ein Schroecksches Schreiben vom 25. November 1697 an den mit dem Cognomen «Aristoteles» versehenen Anatomen wurde bereits verwiesen (18).

Mit Zwingers Berufung endet die Liste der Fehrschen Schweizer Berufungen. Unter seinem Amtsnachfolger Volckamer ist KarL Joseph MüLLER, Stadtphysikus in Zürich, der erste Schweizer Kandidat, wobei die schon erwähnte Gutachterrolle Peyers wichtig gewesen sein dürfte. Als Müller am 24. September 1688 in die Liste der Gesellschaft inskribiert wird, hat seine Aufnahme jedenfalls - wohl im Gefolge der Peyerschen Empfehlung - bereits intern zur Diskussion gestanden, denn sein manu propria ausgefertigter Lebenslauf liegt schon im April 1688 bei Volckamer in Nürnberg vor. Müller notiert darin kurz über seinen Ausbildungsgang:

«Tum variis locis bonarum literarum studia ut vocatur humaniora adolescens excoluit, usque ad annum hujus seculi septuagesimum sextum. Inde Lugduni in Gallia potissium Philosophiae, et Monspelii Medicinae operam dedit, tandemque in urbe Avenionensi quae summo Pontifici paret, Doctoris medici insignia recipit. Anno MDCLXXX posteaquam ad suos in Helvetiam reversus esset Basileam, hinc Argentoratum, ac novissime alio itinere in Italiam se contulit, ad artem medicam exercendam in variis nosocomiis» (5).

Volckamers Präsidialtätigkeit ist nur kurz; nach Müller gelangen in seiner Ära lediglich noch Johann Jаков W vester Samuel Anhorn von Hartwiss zur Aufnahme. Der am 19. Mai 1690 rezipierte Wagner ist Waisenhausarzt in Zürich, Hartwiß wirkt als Archiater in St. Gallen, als ihm das Mitgliedsdiplom zugestellt wird.

\section{Die Schweizer Rezeptionen in der Amtsperiode von Lucas Schroeck}

erreichen zwischen 1694 und 1730 einen zweiten Gipfel: unter dem IV. Präses werden insgesamt 15 Wissenschaftler in die Leopoldina berufen. In chronologischer Folge handelt es sich dabei um JoHAnN Conrad WePFER 
(1657-1711), Johann Georg Joseph Schwaller (geb. 1671), Johann Jakob Franz Vicarius (geb. 1664), Johann Michael Surgant, Johann Jakob Scheuchzer (1672-1733), Johann Heinrich Hottinger (1680 bis 1756), Sebastian Vorster, Johann Jakob Mayr de Mayenburg (1665 bis 1717), Nikolaus Lang (1670-1741), Peter Mangold (geb. 1686), Johann Scheuchzer (1684-1738), Leonhard Hurter (1678-1733), Samuel Battierius und Johann Rudolf Zwinger (1692-1777); die Aufnahme von Moritz Anton Kappeler (1685-1769) wird von Schroeck noch eingeleitet, aber unter seinem Nachfolger Johann Jakob Baier (1677-1735) abgeschlossen.

Wenn Johann Conrad Wepfer am 5. Februar 1694 die Schroecksche Rezeptionsliste einleitet, so mag es sich hier um eine Respektsbezeugung vor dem würdigen Vater gehandelt haben; immerhin ist aber der 1679 in Leiden promovierte Wepfer jun. ein tüchtiger Arzt, dem 1702 das Stadtphysikat von Schaffhausen angetragen wird. An wissenschaftlicher Bedeutung steht der mit Peyer und seinem Schwager Brunner fast gleichaltrige Wepfer jun. allerdings deutlich zurück (22).

Der im folgenden Jahr berufene Johann Georg Joseph Schwaller, Physikus von Solothurn und anschließend Poliater von Basel, kann sich auf eine Empfehlung von Harder beziehen (1). Leider fehlen im Archiv der Leopoldina schriftliche Unterlagen über diese beiden Kandidaten und auch für die nächstfolgenden Mitglieder Vicarius, Surgant, Scheuchzer, Hottinger und Vorster, die zwischen 1697 und 1704 zur Aufnahme kommen. Eine Analyse der für die Miscellanea eingesandten Beiträge läßt erkennen, daß sich bei dem als Poliater Tigurinus fungierenden Johann Heinrich Hottinger der Einfluß des großen Schweizer Enzyklopädisten Johann Jakob Scheuchzer unverkennbar abzeichnet (18); die medizinischen und die pathologisch-anatomischen Arbeiten treten hier hinter der Beschreibung z. B. der helvetischen Eisberge völlig zurück (31).

Erhalten geblieben ist ein Schriftwechsel zwischen Schroeck und dem nächstfolgenden, am 13. Januar 1704 rezipierten Kandidaten JoHANN Jakob Mayr de Mayenburg. Mayr ist aus Schaffhausen gebürtig; sein Curriculum vitae weist ihn als Schüler von Peyer, Screta und Wepfer aus. 1687 in Basel promoviert, nimmt Mayr 1699 eine Archiaterstelle beim Landgrafen von Fürstenberg und beim Herzog von Württemberg an; 1706 wird er vom Kaiser in den Adelsstand erhoben. Schließlich als Archiater in Kassel niedergelassen, scheint Mayr wenig Zeit für eine wissenschaftliche Tätigkeit geblieben zu sein; es heißt hierzu im Bericht der Akademie: 
«Libros vero noster nullos edidit: licet enim saepius nonnullis elaborandis, edendisque scriptis manum admoverat, perficere tamen illi nihil eorum, quae erudite meditabatur laborum negotiorumque et multitudinem et magnitudinem licebat» (6).

Karl Nikolaus Lang ist Arzt in Luzern, als ihn am 17. Oktober 1706 die Aufnahme in die Leopoldina erreicht. Lang hatte zunächst in Rheinfelden und St. Blasien praktiziert und als Militärarzt gedient, bevor er sich in seiner Vaterstadt niederlieB. Er ist bei seiner Berufung bereits als Wissenschaftler auf einer Spezialdisziplin ausgewiesen: seine Arbeiten stellen die frühesten mineralogischen und paläontologischen Untersuchungen in der Schweiz dar (17). In seinem Curriculum vitae teilt Lang mit, daß ihn eigentlich ein Zufall zur Mineralogie geführt hat:

«Casu deprehendit quamplurimas lapidum Figuratorum species ... quorum innumera copia, et admirabilis varietas animum ipsius ita delectavit, ut imposterum omnia a praxi medica libera momenta locorum collectioni et contemplationi destinaverit, tantumque numerum brevi temporis spatio collegit» (7).

Im gleichen Zusammenhang weist dann Lang auf die Anregungen des Barons von Trautmannsdorf hin, eine entsprechende Publikation zusammenzustellen: diese führt dann zur Idea historiae naturalis lapidum figuratorum.

Knapp drei Jahre später wird am 14. September 1708 mit dem Basler Fakultätsassessor Peter Mangold ein junger Arzt in die Gesellschaft berufen, der diese Rezeption wohl ausschließlich einer Empfehlung und nicht den bisher gezeigten Leistungen verdankt, ist er doch gerade erst 1706 in Basel unter Harder promoviert worden. Über seinen Studiengang der Jahre 1703 bis 1706 berichtet er an Schroeck:

«Ab illo tempore Medicinae studio animum applicuit.D.10. Sept.1703 Genevam contendit, linguae Gallicae addiscendae gratia, ubi de meliore nota commendatus Viris clarissimis, D. Clerico, Doct. et Senatori, D. Chenaud, Dn. Butini, Dn. Peyero, tunc Ecclesiae Germanicae pastori, et D.Gautiero, Philosophiae Professori, uberes Scientiae fructus ex frequenti cum ipsis conversatione reportavit. Redux A.1704 d.19. in transitu Lausannae D. Doct. Dasples, et Bernae D.D.König salutavit, ab iisdem amicissime exceptus. Paulo post ... cum ardore Medica tractavit, donec superatis examinibus rigorosis pro Supremis in Medicina honoribus Dissertationem de sex rebus non naturalibus feliciter defendit d.29. April.1706.» (8).

Der Schriftverkehr der nächstfolgenden Leopoldina-Mitglieder - das sind Johann Scheuchzer, Leonhard Hurter, Samuel Battierius und Johann Rudolf Zwinger - ist im Briefarchiv der Gesellschaft nicht mehr vorhanden; Schroeck nimmt diese vier Schweizer zwischen 1708 und 1723 in die Sozietät auf. Dagegen ist ein Bericht über seinen letzten Schweizer 
Aufnahmekandidaten erhalten geblieben: Moritz Anton Kappeler ist für die Mitgliedschaft bereits vorgesehen, als bei Schroecks Ableben die Formalitäten noch nicht abgeschlossen sind; so rangiert der am 1. Juni 1730 inskribierte Kappeler bereits in der Ära seines Nachfolgers Baier.

Über Moritz Anton Kappeler liegt ein ausführliches Curriculum vitae vor, das aber erst nach 1769 ausgefertigt wurde. Das Akademiesekretariat überträgt den lateinischen Text ins Deutsche; der ausführlich gehaltene Bericht liefert über den Arztsohn aus Willichau bei Luzern präzise Auskünfte. Demnach ist der 1706 in Pont-à-Mousson promovierte Kappeler zunächst kaiserlicher Truppenarzt in Italien gewesen, wobei er sich gleichzeitig als Ingenieur auszeichnete. In die Heimat zurückgekehrt, widmet sich Kappeler hier vor allem der kristallographischen Forschung; wegen seiner vorzüglichen Arbeit beruft ihn Isaac Newton (1643-1727) schon im Jahre 1726 in die königlich-britannische Akademie der Wissenschaften; vier Jahre später folgt die Leopoldina.

Über Kappelers postgraduale Tätigkeit heißt es in dem genannten Bericht:

«Unerachtet seiner vielen Geschäfte, unterließ er doch nicht, sich dem Studieren auf das Geflißenste zu ergeben. Neben der Mathematic, Medicin, Botanic, Algebra, Optica, Geometria, Hydraulica, Mathematica, Historia, Geographia etc.legte er sich auch vornehmlich mit großem Fleiß auf die Physic und Natur-Historie; das Studium der natürlichen Dinge, so bereits beßer betrieben zu werden begunte, war ihm sonderbahr angemeßen; und ja mehr er sich darin vertiefte, je mehr Annehmlichkeiten fand er darinnen, und wurde begierig sie zu ergründen. In der Botanic, dieser den Medicis so unentbehrlichen Wißenschafft, hielt er sich an das Systema des Tourneforts, welcher am ersten die ... Pflanzen, in gewiße Claßen, um sie dem Gedächtniß zu erleichtern, brachte. Bey der Ausübung seiner Medicin war er meißtentheils darauf bedacht, wie und auf was Weis, auch in den schwersten Zufällen und Kranckheiten mit leichtester Mühe und geringen Mitteln denselbigen Inhalt gethan, und die vorige erwünschte Gesundheit denen Patienten wieder möchte hergestellet werden.

Die Crystall-Gruben so man 1724 auf dem Grimselberg in dem Canton Bern, entdeckte, machte alle Natur-Forscher aufmerksahm: H. Cappeler aber wolte die Wahrheit von dem, was von der beständigen Figur dieser leuchtenden Cörper gemeldet wurde, selbsi einsehen; Er begab sich selbst an diesen Ort, und befand es würcklich also, daß unter allen Crystallen, keiner dem andern in allem durchaus ähnlich sey, ob sie gleich alle nach einem gleichen Plan gestaltet sind. Dieses gab ihm Anlaß nach seiner Zurücklkunft an seiner Chrystallographia zu arbeiten, so er unter dem Namen Adumbratio Chrystallographiae Historicae physicae medicae ankündigte: Allein es kamen so viele Hinderniße wegen überhäufften Geschäfften dazu, das davon nur das vierte Capitel dieses Wercks unter dem Titel: Prodromus Chrystallographiae de Chrystallis improprie Sic. dictis Commentarium, 4. Lucern. 1723. cum Figuris herausgekommen; zu welchen und andern Kuperstichen, in seinen Wercken er die Riße selbst verfertiget. Von diesem Prodromo ist auch ein Auszug den Englischen Transactions Philosophiques einverleibt worden, und hat 
ihm einen Platz unter den Mitgliedern, bey der Königl.Brittannischen Gesellschaft der Wißenschaften 1726 zuwege gebracht, da eben der große Newton Präsident derselbigen war. Ein guter Theil des noch übrigen Manuscripts liegt in den Händen eines großen Freundes des Verstorbenen, und zu dem übrigen ist ihm von dem Verstorbenen auch Hoffnung gemacht, dabey aber zugleich gemeldet worden, daß wegen der Menge von Verbeßerungen, Zusätzen und Durchstreichungen, und weil sie mit zitternder Hand geschrieben worden, selbige schwerlich zu lesen seyn dürfte.

Die genaueste Freundschaft, so er mit dem Schweizerischen Plinio, dem berühmten Herrn Doctor und Chorherr Johann Jakob Scheuchzer, viele Jahre lang unterhalten, und der, nebst Woodward und Luid in Engeland, auch H.Doctor Lang, zu Lucern, das Studium Lithographiae sehr beförderte, gaben unserem H.Cappeler Gelegenheit, ihm viele gelehrte Gedanken hierüber von Zeit zu Zeit mitzutheilen, davon nur zum Beweiß mag angeführt werden das gelehrte Brief: De Entrochis et Belemnitis, den der berühmte Naturforscher Kleinius zu Danzig als eine Vorrede seinem Nomenclator de Lapidibus Figuratis Ao. 1740 zu Danzig herausgekommen, vorgesetzet hat; und gestehet, daß in demselbigen die Entstehungs-Arten dieser versteinerten Thiere am besten bis dahin aufgekläret worden» (9).

Kappelers weiterer Lebensweg steht hier nicht zur Abhandlung an. Für die Leopoldina ist er stets aktiv gewesen, so daß ihm der Übersetzer des Curriculum vitae bescheinigen kann: «und findet man in den gelehrten Abhandlungen dieser Gesellschaft verschiedene lesenswerte Dissertationen von ihm».

Die Inskription von Kappeler bleibt die einzige von Baier für das Schweizer Territorium. Nach seinem Ableben übernimmt Andreas Elias Büchner (1701-1769), der unter dem V. Praeses als Adjunkt fungiert hatte, die Geschäftsführung der Gesellschaft.

\section{Die Präsidialperiode von Andreas Elias Büchner}

verlagert den Sitz der Leopoldina zunächst nach Erfurt und dann ab 1745 nach Halle, als Büchner einem Ruf an die Academia Fridericiana folgt. Bemerkenswert ist für die Büchnersche Ära, daß im Gegensatz zu den ständig anwachsenden Vokationen aus Ungarn (28) und Dänemark-Schweden (29) die Schweizer Rezeptionen jetzt deutlich stagnieren. Eine genauere Analyse läßt zudem erkennen, daß es sich bei den unter Büchner berufenen helvetischen Wissenschaftlern nunmehr vielfach um Persönlichkeiten handelt, die außerhalb der Landesgrenzen ihrer Heimat wirken. Vom Geburtsort oder von ihrer Tätigkeit her müssen für die Ära des VI. Präsidenten die folgenden Namen genannt werden: Johand JaKoв Ritter (1714-1780), Johann Jakob Huber (1707-1778), Johann Gesner (1709-1790), AlbRecht von Haller (1708-1777), Rudolf von Schwachheim, Lorenz Spengler, Johann Ulrich Bilguer (1720-1796), Johann Caspar Sulzer 
(1716-1799), Charles Bonnet (1720-1793), Theophil Sigismund Gruner (1718-1778) und Wilhelm Ritsch (geb. 1728).

Die Büchnersche Aufnahmeliste Schweizer Kandidaten beginnt mit Johann Jakob Ritter aus Bern und Johann Jakob Huber aus Basel. Beide sind sie Haller-Schüler: die Parallele zu Wepfer bietet sich an, denn auch jetzt werden die einstigen Adlati noch vor dem Lehrer rezipiert. Ritters Aufnahme erfolgt am 25. Juni 1740 und damit zu einem Zeitpunkt, als der Berner Mediziner die Nachfolge des vom heimatlichen Amt abgegangenen Haller angetreten hat. Ritter berichtet hierüber an Büchner:

«Mense Martio 1739. ex Decreto supremi Magistratus Successor Halleri in Professione Anatomica constitutus, Collegia Anatomica privata publicaque legit, demonstrationes publicas continuavit»... (10).

Schon 1740 folgt Ritter aber einem Ruf als Archiater nach Hessen-Homburg und läßt sich schließlich später in Ober-Peilau bei Nimptsch in Schlesien nieder. Das Briefarchiv der Leopoldina weist seinen über vier Jahrzehnte fortbestehenden Kontakt mit der Gesellschaft aus; dabei versucht Ritter wiederholt, die Akademie für profilierte Schweizer Landsleute zu interessieren: offenbar mit geringem Erfolg, wie 1771 sein vergeblicher Einsatz für Johann Georg Zimmermann (1728-1795) und Simon André Tissot (1728-1797) erkennen läßt. Im gleichen Schreiben empfiehlt er außerdem einen (namentlich nicht genannten) Zürcher Kollegen und legt dessen (verlorengegangenes) Curriculum vitae vor. Da eine Rezeption aus Zürich für die Folgezeit nicht nachweisbar ist, hat man offensichtlich Ritters Vorschläge generell übergangen. Das bestätigt das mangelhafte Engagement dieser Jahre, wie es auch aus den Zeilen von Ritter spricht, als er sich an Büchners Nachfolger Ferdinand Jakob Baier (1707-1788) wendet:

«Da ich in dem Catalogo receptorum von 1765-1770 meine Landsleute zwey verdienstvolle Männer; den Königl.Leib Medicum H.D.Zimmermann aus Brugg, und den berühmten Practicum H.D. Tissot noch nicht gefunden habe, so zweifle billig, ob sie unter dem Praesidiat des seel. H.D. Büchners sind in die Academie aufgenommen worden. Zweifelsohne werden Ew. Hochwohlgeb. sich am Antritt dero Praesidiats schon, nach Dero beiwohnenden Eifer, Verdienste $z u$ befördern, an diese zwey würdigen Männer gedacht haben, ohne daß ich nöthig habe selbige bey Ihnen in Andenken zu bringen» (10).

Der im Alter schwerkranke Ritter hält auch dann Verbindung zur Akademie, als er für den Schriftverkehr auf fremde Hilfe angewiesen ist. So läßt er am 20. Juli 1779 von Ober-Peilau aus an das inzwischen in Ansbach stationierte Sekretariat berichten: 
«Durch den Schlag ist die Zunge gelähmt worden, so daß das Reden schwer fällt. Das Judicium hat großen Schaden gelitten. Die mathematischen Wißenschaften habe ganz verlohren, so daß ich auch nicht bis auf 12 addiren oder subtrahiren kann; hingegen derer vergangenen historischen Dingen ist das Gedächtniß ganz frisch. Hingegen ist die Einbildungs-Kraft zu meiner großen Beschwerde äußerst lebhaft.

Die getroffenen Glieder sind nicht lahm, sondern schmerzhaft steif, und die Finger der rechten Hand zusammengezogen. Der Rückgrad paralitisch, samt dem Intestino recto, so daß die Solutio alvi nicht anders als per Clysmata erhalten werden muß.

Ohngeachtet einer sehr beweglichen Lebensart, habe ich seit 15 Jahren eine tägliche Abnahme im Gehen verspüret, die ohngeachtet des Spazirengehens und Fahrens beständig zugenommen, daß schon seit 8 Jahren, außer Stand war allein zu gehen, und mich durch zwey Diener unter den Arm führen, endlich seit 6 Jahren tragen laßen, ohne Paralysis. Im Jahr 1778 war ich außer Stand auch so gar das Tragen ferner auszustehen; und endlich dieses Jahr so gar apoplectisch» (10).

Ritters letztes Schreiben an die Leopoldina geht am 23. Dezember 1779 ab - dann endet die langjährige Korrespondenz.

Den Haller-Schüler JoHanN Jаков HubER nimmt Büchner noch von Erfurt aus am 28. Januar 1741 in die Leopoldina auf. Der auf einer Professur in Kassel wirkende Basler Mediziner ist durch Arbeiten über das Nervensystem, das Foramen ovale, die weiblichen Genitalorgane sowie durch die Beschreibung von Muskelvarietäten besonders als Anatom hervorgetreten.

Büchner ist bereits nach Halle übergesiedelt, als er am 4. September 1746 den Zürcher Ordinarius Johann Gesner in die Leopoldina beruft. Gesner bedankt sich am 4. April 1748 recht herzlich und gibt dabei seiner Freude Ausdruck, daß ihm das Cognomen «Acarnan» zugebilligt wurde, das vor ihm der von ihm hochverehrte Johann Jakob Scheuchzer getragen hatte. Gesner steckt gerade voller Arbeit für die ihm übertragene Direktion der Schweizer Physikalischen Gesellschaft; er entschuldigt sich daher, daß er dem Schreiben keine größere Abhandlung zwecks Publikation beilegen kann:

«Ich würde auch dermahls einige Physicalische und Medicinische Beobachtungen beygefüget haben, wenn nicht die dermahlige aufhebende Rectoratsstelle bey unserem Gymnasio, und die Einrichtung einer seit einem Jahr unter meinem Subsidio angefangenen Physicalischen Gesellschaft bey meiner zärtlichen Gesundheit, mir viele Stunden entzogen hätten» (11).

Im gleichen Schreiben erwähnt Gesner einen seiner früheren Hörer, der nun bei Büchner in Halle studiert: es ist Johann Heinrich Tschudi aus Glarus, der dann am 17. April 1750 unter Büchner pro gradu doctoris medicinae disputieren wird (26): 
«Herr H.Tschudi Med.Stud.auß einer der ansehnlichsten Familien des uns benachbarten und mit verbündeten löblichen Cantons Glarus, der hier einige Zeit bey H.D. und Stadt Arzt Landolt einigen exercitiis beygewohnet, auch die Demonstrationes auf unserm Theatro Anatomico mit angesehen hat, bey mir auch einige Vorlesungen über die Naturlehre, Botanic und Boerhaavens Physiologie angehöret» (11).

Die von Gesner dirigierte Physikalische Gesellschaft wird auch in dem bereits zitierten Curriculum vitae von Kappeler erwähnt:

«Die 1762 auf das Neue belebte Physicalische Gesellschaft allhier, nahm auch, da sie auswärtige Ehren Mitglieder zuerst aufgenommen, ihn am 9.Jan. unter Praesidio H.Doctor und Chorherr Geßners, unter dieselbige auf, und ladete ihn ein, mit seinen Einsichten zum Nutzen des Menschlichen Geschlechts ihro beyzustehen. Welcher Sammlungen dritten Bands auch einverleibt worden seine kurtze Beschreibung einer neuen Zubereitung oder Saturation der KrebsAugen, und des Gebrauchs derselbigen, in verschiedenen sonderbar hitzigen Kranckheiten » (9).

Der am 10. Januar 1750 rezipierte Albrecht von Haller bedarf hier keiner biographischen Erörterung - der Hinweis, daß der Lehrer erst nach seinen Schülern zur Berufung kam, wurde schon gegeben. Hallers weltweite wissenschaftliche Bedeutung erstreckt sich auf zahlreiche Fachdisziplinen $(23,38)$. Das Leopoldina-Archiv enthält ein ausführliches - allerdings nicht von ihm selbst niedergeschriebenes - Curriculum vitae für die Jahre 1753-1777; es schließt mit mehreren Zusammenstellungen wie «Opera praecipua» sowie den Hinweis auf die zuständigen Periodika («l'on trouve aussi de ses ouvrages dans les Recueils suivants») und die Rubrik «Des Prefaces ouvrages suivans» (12).

Der nächstfolgende Kandidat ist Rudolf von Schwachнeim, hallescher Doktorand von 1757. Der aus Vaduz gebürtige Arzt interessiert hier nur randständig; seine Rezeption erfolgt am 3. März 1759. Über ihn sowie über Lorenz Spengler (aufgenommen am 12. Oktober 1761) liegen hier keine näheren Angaben vor. Das Curriculum vitae des in preußischen Militärdiensten berühmt gewordenen Johann Ulrich Bilguer aus Chur (14) ist dagegen erhalten geblieben; es wurde von uns anläßlich der 250. Wiederkehr seines Geburtstages veröffentlicht (25), wobei gleichzeitig die im 18 . Jahrhundert sehr enge Anlehnung der studierenden Jugend Graubündens an den Pietismus hallescher Prägung aufgezeigt werden konnte (27).

Johann CASpar Sulzer aus Winterthur ist schon fast fünfzehn Jahre nicht mehr in seiner Schweizer Heimat ansässig, als ihn am 10. Januar 1763 die Berufung in die Leopoldina erreicht; er war 1749 als herzoglicher Leibarzt an den Gothaer Hof gekommen und hatte hier die Modernisierung des Medizinalwesens im Doppelherzogtum Gotha-Altenburg besorgt (33, 34). 
Sulzer ist übrigens nicht der erste Arzt, der aus Winterthur gebürtig ist und zur Akademie in Beziehung tritt: schon Johann Heinrich Hegner (1646-1696) hatte in den Miscellanea publiziert, war aber nicht zur offiziellen Mitgliedschaft gelangt. Die Korrespondenz Sulzers mit der Leopoldina ist dort nicht mehr erhalten, auch nicht diejenige des gemeinsam mit ihm rezipierten Charles Bonnet aus Genf und des Berner Naturwissenschaftlers Theophil Sigismund Gruner, der am 12. Juni 1766 sein Diplom ausgestellt erhält. Der letzte in diesem Zusammenhang zu nennende Büchner-Kandidat dagegen - es ist der am 21. Oktober 1766 berufene sächsischpolnische Militärarzt Wilhelm Ritsch - ist in seinem frühen Werde- und Ausbildungsgang durch seinen eingereichten und erhalten gebliebenen Lebenslauf genau verfolgbar. Seine Nominierung an dieser Stelle basiert auf der von ihm selbst gemachten Mitteilung, daß er vorübergehend in Genf bei dem bekannten Pockeninokulator Théodore Tronchin (1709-1761) gearbeitet hat:

«Ex Italia ad felicem rem publicam Genevensem advolavi, quo in loco celeberrimus Vir Tronchin varia ratione suam benevolentiam mihi demonstravit, et egregie de meis meritus fuit studiis» (14).

\section{Die auf Büchner folgenden Akademiepräsidenten}

haben in den letzten Dezennien des 18. Jahrhunderts die einst so engen Kontakte in die Schweiz kaum intensiviert; die Neuaufnahmen bleiben auch jetzt spärlich. Unter dem bis 1788 amtierenden VII. Praeses Ferdinand Jakob Baier gelangt Amadeus Emmanuel von Haller am 24. Februar 1778 zur Rezeption; der Aufnahme war ein Briefwechsel vorausgegangen, in welchem es sich um den wissenschaftlichen Nachlaß des berühmten Vaters und um ein würdiges Pro-Memoria handelt. Haller jun. schreibt am 23. Januar 1778 an Baier:

«Die gütige Aufnahme der Beyträge zu meines sel.Vatters Lebensbeschreibung, ist mir ein schätzbarer Beweiß dero Wohlwollens, deßen Beybehaltung mir äußerst angenehm ist. Der vorhandenen Arbeit zu Ehren meines sel.Vatiers sehe ich mit Ungeduld entgegen, und bitte ergebenst um einige Exemplare derselben, wann sie abgedruckt seyn wird.

Die Historia stirpium Helvetiarum werde ich so bald möglich die Ehre haben als ein geringes Kennzeichen meiner Hochachtung für die Kayserl. Gesellschafi der Naturforscher zu übersenden. Sehr schmeichelhaft wär es für mich ein Mitglied derselben zu seyn, wenn ich dieser Ehre würdig wäre. Die zwey andern begehrten Bücher sind noch nicht heraus» (15). 
Für die auf Eigenantrag nunmehr durchgeführte Inskription bedankt sich Haller jun. am 14. März 1778 und schreibt 1780 an Baier, der ihn für die Leopoldina um ein Exemplar der Hallerschen Physiologie gebeten hatte:

«Ich werde alle Sorge tragen daß die Bibliothek der Kayserl.Gesellschaft mit meines sel. Vatters Physiologie versehen werde. Ob es gegen Bezahlung oder sonst geschehen werde, kan ich noch itzo nicht bestimmen, diß kommt auf meine Miterben an, welche die Exemplare dem Verleger bezahlen müßen. Ich werde aber mein möglichstes thun, daß dieses Werck ohnentgeltlich Ew. Hochwohlgeb. eingehändiget werde» (15).

Unter Baier wird am 16. August 1781 auch dem Schweden JoнAnN Jaков Ferber (1743-1789) die Ehre der Aufnahme zuteil. Der wechselhafte Lebensweg dieses als Mineraloge hervorgetretenen Wissenschaftlers endet auf einer Studienreise in die Schweiz: nach schwerer Krankheit stirbt Ferber in Bern und wird an der Seite Albrecht von Hallers beigesetzt (24).

Das hallesche Leopoldina-Archiv enthält über die drei letzten Schweizer Wissenschaftler, die zum Jahrhundertende zur Aufnahme kommen - es sind Johann Heinrich Rahn (1749-1812), Paul Usteri (1767-1831) und Franz Ferdinand Will - keine Unterlagen mehr über einen zweifellos vorhanden gewesenen Schriftwechsel. Die noch zur Verfügung stehenden Originalien der übrigen und im Rahmen der vorliegenden Abhandlung aufgeführten Mitglieder schienen uns aber interessant genug, um sie - wenn an dieser Stelle auch nur fragmentarisch - einer breiteren Leserschaft vorzustellen. Sie dürften sowohl für biographische Ergänzungen und Richtigstellungen als auch zur Interpretation größerer Sachzusammenhänge von Bedeutung sein und sollten daher eine vollständige Auswertung erfahren. Der erhalten gebliebene Schriftwechsel weist jedenfalls nachdrücklich aus, welche Bedeutung den Wissenschaftskontakten nach der Schweiz in bestimmten Zeitabschnitten des 17. und 18. Jahrhunderts beigemessen wurde.

\section{Quellen und Literatur}

(Abkürzung: A.A.N.L. = Archiv der Deutschen Akademie der Naturforscher Leopoldina).

1 A.A.N.L. Halle, Briefarchiv Nr. 94.

2 A.A.N.L. Halle, Briefarchiv Nr. 96.

3 A.A.N.L.Halle, Briefarchiv Nr. 100.

4 A.A.N.L.Halle, Briefarchiv Nr. 105.

5 A.A.N.L.Halle, Briefarchiv Nr. 160.

6 A.A.N.L. Halle, Briefarchiv Nr. 261. 
7 A.A.N.L.Halle, Briefarchiv Nr. 267.

8 A.A.N.L.Halle, Briefarchiv Nr. 282.

9 A.A.N.L. Halle, Briefarchiv Nr.411.

10 A.A.N.L. Halle, Briefarchiv Nr. 502.

11 A.A.N.L.Halle, Briefarchiv Nr.547.

12 A.A.N.L.Halle, Briefarchiv Nr. 560.

13 A.A.N.L. Halle, Briefarchiv Nr.649.

14 A.A.N.L. Halle, Briefarchiv Nr. 689.

15 A.A.N.L.Halle, Briefarchiv Nr. 821.

\section{Literatur}

16 Brunner C. und W.v.Muralt, Aus den Briefen hervorragender Schweizer Ärzte des 17. Jahrhunderts, Basel 1919.

17 Buess H., Schweizer Ärzte als Forscher, Entdecker und Erfinder, Basel 1945.

18 Buess H., Der Beitrag der Schweizer Ärzte zu den «Miscellanea curiosa» der Deutschen Akademie der Naturforscher, Sudhoffs Arch.37 (1953) 1-22.

19 Duka N., Ärztliche Beziehungen zwischen der Schweiz und der Slowakei vom 16. bis zum Ende des 18. Jahrhunderts, Gesnerus 1/2 (1965) 68-84.

20 Fischer H., Johann Jakob Wepfer (1620-1695), Mitteilungen der Naturforschenden Gesellschaft Schaffhausen, Heft IX (1931) 93-201.

21 Fischer H., Briefe Johann Jakob Wepfers (1620-1695) an seinen Sohn Johann Conrad (1657-1711), Studiosus Medicinae zu Basel und Leyden, Veröffentlichung der Schweizerischen Gesellschaft für Geschichte der Medizin und der Naturwissenschaften, Band XIII (1943) 5-68.

22 Fischer H., Beziehungen der Schaffhauser Ärzteschule des 17.Jahrhunderts zur Deutschen Akademie der Naturforscher, Mitteilungen der Naturforschenden Gesellschaft Schaffhausen, Heft XXVIII (1963/67) 1-28.

23 Hintzsche E., Albrecht von Hallers Bedeutung für die Geschichte der Medizin, Verhandlungen des XIX. Internationalen Kongresses für Geschichte der Medizin, Basel 1964, Basel/New York 1966.

24 Kaiser W.und H.Krosch, Hallesche Doktoranden als Mitglieder der Academia Imperialis Leopoldino-Carolina Naturae Curiosorum, Wiss.Z. Univ. Halle (Math.-naturw.) XVI (1967) 603-64.4.

25 KaISER W., Johann Ulrich Bilguer (1720-1796) und die Medizinische Fakultät Halle, Gesnerus 27 (1970) 85-95.

26 Kaiser W.und W.Piechocki, Schweizer Medizinstudenten und Ärzte als Absolventen der Medizinischen Fakultät Halle, Gesnerus 26 (1969) 189-212.

27 Kaiser W.und W.Piechоскі, Graubündner Studenten des 18. Jahrhunderts an der Universität Halle, in Äskulap in Graubünden, S.647-679, Chur 1970.

28 KaISER W.und A.VöLKer, Ungarländische Leopoldina-Mitglieder des 18. Jahrhunderts und ihre Korrespondenz mit den Akademiepräsidenten, Comm. Hist.Med. (im Erscheinen). 
29 KAISER W.und A.VöLKer, Skandinavische Leopoldina-Mitglieder des 18. Jahrhunderts und ihre Korrespondenz mit den Akademiepräsidenten, Sydsvenska Medicinhist., Sällskapet (1972) 94-108.

30 Mani N., Das «Buch über die Wiederkäuer» («Merycologia»), eine der geschichtlichen Grundlagen der heutigen Haustierphysiologie, Gesnerus 8 (1951) 123-137.

31 Niggli P., Die Krystallogia von Johann Heinrich Hottinger, Veröffentlichung der Schweizerischen Gesellschaft für Geschichte der Medizin und der Naturwissenschaften, Band XIV, Aarau 1946.

32 Nigst H., Das Anatomische Werk Johann Jakob Wepfers (1620-1695), Veröffentlichung der Schweizerischen Gesellschaft für Geschichte der Medizin und Naturwissenschaften, Band XVI (194:7) 1-88.

33 Johann Caspar Sulzer $\left({ }^{*} 1716\right.$ zu Winterthur, $\uparrow 1799$ zu Gotha $)$ und sein Sohn Friedrich Gabriel Sulzer (*1749 zu Gotha, $\dagger 1830$ zu Altenburg). Zwei hervorragende Mediziner schweizerischer Herkunft im goethezeitlichen Thüringen, Verhandlungen des XIX. Internationalen Kongresses für Geschichte der Medizin, Basel 1964, S.416-419, Basel/ New York 1966.

34. Petzsch H., Der Humanmediziner Dr. F. G. Sulzer (1749-1830) und sein Ronneburger Vieharzeneiinstitut, Dtsch.med.J.20 (1969) 123-128.

35 Peyer B., Die biologischen Arbeiten des Arztes Johannes von Muralt (1645-1733), Thayngen 1946.

36 Peyer B., Der Arzt Johann Conrad Peyer, Zürich 1932.

37 Peyer B.und H. Peyer, Bildnis und Siegel des Arztes Johann Conrad Peyer, Schaffhausen 1943.

38 SсHÄR R., Albrecht von Hallers neue anatomisch-physiologische Befunde und ihre heutige Gültigkeit, Inaug.-Diss., Bern 1958.

39 Schwertz F., Die Heilmittel in den Schriften des Schaff hauser Arztes Heinrich Skreta über die Lagersucht, in Festschrift für Dr.J.Brodbeck-Sandreuter, Basel 194.2.

40 Zimmermann O.C., Die erste Beschreibung von Symptomen des experimentellen Pankreas-Diabetes durch den Schweizer Johann Conrad Brunner (1653-1727), Gesnerus 2 (1945) 109-130. 